Fecha de recepción: octubre 2021 Fecha de aprobación: noviembre 2021 Fecha publicación: diciembre 2021

\section{Moda o ¿cómo interactuar con las generaciones jóvenes para crear marca?}

Joan-Francesc Fondevila-Gascón ${ }^{(1)}$, Pedro Mir-Bernal ${ }^{(2)}$, Teresa SádabaGarraza $^{(3)}$ y Fátima Vila-Márquez ${ }^{(4)}$

Resumen: Los millennials están ligados a la tecnología y a las nuevas formas de comunicación. A través de las redes sociales, los millennials se relacionan con las principales marcas multiplicando la interacción y evolucionando constantemente las percepciones con estas, hecho que contrasta con otras generaciones que no podían relacionarse de forma tan rápida y frecuente con las marcas. Mediante el estudio de las percepciones que tienen los millennials sobre Zara, H\&M, Louis Vuitton y Gucci se busca conocer el posicionamiento de cada una de estas marcas entre el target seleccionado, los millennials españoles, así como también comprender la posición que ocupan entre los millennials. Se observa una asociación moderada entre atributos y marca y un alto grado de reconocimiento y notoriedad.

Palabras clave: Moda - millennials - lujo - percepción - posicionamiento - tecnología marcas - comunicación - redes sociales - identidad.

[Resúmenes en inglés y portugués en las páginas 31-32]

(1) Joan Francesc Fondevila Gascón es Doctor en Periodismo con Sobresaliente Cum Laude por la Universitat Autònoma de Barcelona, Primer Premio Redes de Telecomunicaciones por Cable, Primer Premio Connexió y dos veces Primer Premio de Investigación Prensa Comarcal. Su línea de investigación gira en torno a periodismo digital, telecomunicaciones y banda ancha, Internet, HbbTV y redes sociales. El Dr. Fondevila ha publicado más de un centenar de artículos en revistas de impacto científico y varias obras monográficas. Está acreditado como Profesor Titular de Universidad por la ANECA y Profesor Agregado por la AQU, y posee un sexenio de investigación reconocido por la AQU. Actualmente, imparte docencia en la Universitat Pompeu Fabra, URL, EU Mediterrani (UdG), UOC, UB, UAB, EAE (UPC) y Euncet (UPC). El Dr. Fondevila es director del Centro de Estudios sobre el Cable (CECABLE). Blanquerna-Universitat Ramon Llull. Cesine, Euncet-UPC, EAE Business School. Autor del blog científico http://www.telecomunicacionesyperiodismo. com/, líder internacional en sus áreas de conocimiento.

${ }^{(2)}$ Pedro Mir es Doctor en Marketing y Comunicación por la Universidad San Pablo CEU. Ha desarrollado su formación en diversas universidades internacionales como Fudan Shanghai University en China, Case Weatherhead Cleveland-Ohio (USA) y comportamiento del consumidor en el MIT de Boston (Massachusetts USA). En los últimos veinte años 
ha desempeñado puestos ejecutivos en el área de Marketing e Innovación en Barna Consulting Group, Danone, Nutrexpa o la suiza Lindt\&Sprüngli. Es profesor visitante en la Universidad Nikolaus Copernicus (Polonia) y del Boston College de Boston y Profesor en la Universidad de Navarra y Universidad Pompeu Fabra.

(3) Teresa Sádaba es Doctora en Periodismo por la Universidad de Navarra y profesora titular de Periodismo en la Facultad de Comunicación de la Universidad de Navarra. Desde 2010 es Directora de ISEM Fashion Business School.

${ }^{(4)}$ Fátima Vila es Doctora Cum Laude en Comunicación por la Universidad Complutense, profesora en EAE Business School y Universidad de Barcelona y periodista especializada en comunicación estratégica, contenidos y marketing online, redacción de contenidos online y offline, marketing político y comunicación.

\section{Marco teórico}

\subsection{Marcas y posicionamiento}

Una marca era de origen meramente un nombre o un diseño pensado para identificar y distinguir los bienes o servicios de un vendedor de otro. Es más, según la American Marketing Association, se define marca como un nombre, término, signo, símbolo, diseño o combinación que identifica productos y servicios y los diferencia de los de la competencia (American Marketing Association, 2017).

Numerosas empresas tratan a la marca como un símbolo. Otras desarrollan un conjunto de estrategias de branding para darle un significado mucho más profundo y complejo, a crear, mantener, proteger y mejorar (Kotler, 2000).

Podemos definir una marca como una representación de un conjunto de características percibidas por los consumidores. Estas características son el producto o servicio, la experiencia de compra, valores y otras características percibidas y asociadas a la marca por el consumidor (Kapferer, 2008).

Según Kotler (2001), las marcas pueden ser percibidas por sus consumidores o público objetivo a través de seis dimensiones. Las empresas desean implantar en la mente del consumidor una percepción positiva de la marca. No es suficiente con crear un producto o servicio hecho a medida para el público objetivo, puesto que los consumidores se afanan en buscar otros beneficios. Con frecuencia no se adquieren productos concretos, sino sensaciones o percepciones, experiencias y sentimientos. Además, la esencia de la marca reside en sus valores, su cultura y su personalidad.

Al diseñar una estrategia de branding las empresas acostumbrar a considerar como dimensiones los atributos de marca, es decir los indicadores con los que se asocia la marca, los beneficios que proporciona, los valores, la cultura de marca, la cual si transmite o repre- 
senta una cultura determinada, la personalidad y el usuario que indica, a qué segmento se dirige.

De hecho, la competencia no se halla entre los productos que emergen de las fábricas, sino del agregado a la producción en forma de un conjunto de factores como el asesoramiento al cliente, el packaging y entrega, los servicios, la publicidad o la financiación. Las marcas van más allá del nombre, y se concentran en ofrecer un plus al cliente (Levitt, 2001).

Los consumidores eligen comprar productos de marcas con las que se sienten asociados. Para atraer al público objetivo, es fundamental crear una personalidad de marca que le resulte atractiva o con la que se sienta identificado. Los consumidores compran productos o servicios que les permiten expresarse a través de ellos. De esta manera, la marca es solo diferenciadora de la competencia, sino que también atrae clientes con preferencias asociadas con la personalidad de la marca (Aaker, 1997 y 2011).

Así, la marca es un activo de las empresas, ya que la percepción de marca que tienen los consumidores acaba por devenir el atributo más valorado del producto o servicio ofrecido. La habilidad para simplificar las decisiones que tienen las marcas permite a las empresas diferenciarse de la competencia y fidelizar al consumidor (Keller, 1998). Es muy necesario que en un entorno tan competitivo como el que hay hoy en día, las marcas se preocupen por convertir su marca en un activo.

Keller (1998) analiza la importancia de construir y nutrir una marca desde dos perspectivas, la del consumidor y la del empresario. Desde el punto de vista del consumidor, una clave es la identificación de la fuente de un producto. Si los consumidores reconocen y conocen la marca, el proceso de toma de decisión se simplifica. En cuanto a la reducción del riesgo, las marcas limitan el peligro de que una adquisición no aporte beneficios, gracias a experiencias pretéritas. En el entorno de comercio electrónico, que requiere un gran flujo de contenidos o cloud journalism (Fondevila-Gascón, 2010) en el contexto de la Sociedad de la Banda Ancha (Fondevila-Gascón, 2013), el consumidor intenta minimizar el riesgo en business to consumer (B2C) o business to business (B2B). Errores de entrega reducen las puntuaciones de experiencia de usuario y ponen en jaque la reputación de la empresa compradora y sus recursos financieros.

Otro factor que valora el consumidor es la reducción del coste de búsqueda. Las marcas permiten a los consumidores reducir costes internos los cuales facilitan la decisión para elegir el producto o servicio y externos que deben dedicar una menor cantidad de tiempo a la búsqueda del producto adecuado. Ello depende de la información de la marca a disposición del cliente, que permite al consumidor crear expectativas sobre el producto o servicio que desea adquirir.

En cuanto a las promesas, al vínculo o al pacto con la marca, es un indicador especialmente sensible en innovaciones como el HbbTV, Hybrid broadcast broadband TV, (Fondevila-Gascón, Botey-López y Rom-Rodríguez, 2017).

Los valores, la cultura y la personalidad de la marca permiten a las empresas establecer una relación marca-consumidor en la que los consumidores se comprometen a ser leales mientras la marca cumpla con las expectativas respecto al producto, precio, distribución y otras variables que le aporten una utilidad o beneficio al cliente. Si el consumidor deja de percibir las ventajas de comprar un producto o servicio o no obtiene ninguna utilidad de su compra, dejará de ser leal a la marca. 
Otro factor es el valor simbólico, según el cual los consumidores pueden desarrollar una gran devoción por ciertas marcas que les hacen sentirse mejor con ellos mismos porque les aportan características que ansían, como podría ser un estatus social alto, en la zona elevada de la pirámide Maslowiana.

Finalmente, el consumidor se ciñe a señales de calidad. Así, el consumidor elegirá comprar los productos o servicios dependiendo de la calidad que esté buscando, que dependerá de otros factores como por ejemplo el precio o la seguridad.

Como beneficios para la empresa, la marca protege características únicas. Legislativamente, se protegen los atributos del producto o servicio que componen la marca. Para proteger la marca, ésta debe ser inscrita en el registro mercantil. Los procesos de producción pueden ser patentados. También se puede proteger legalmente el diseño. Si los anteriores aspectos no están protegidos por la ley, la marca pierde valor como activo de la empresa. La marca es un síntoma de calidad para los clientes satisfechos. Invertir en la marca conduce a crear relaciones marca-producto que conducen a más fidelidad del cliente, más ingresos y una posición más elevada y estable en el mercado para la empresa.

Asimismo, los productos consiguen asociaciones únicas gracias a las marcas y proporcionan una ventaja competitiva, amén de un elemento de seguridad a la empresa a la que pertenece. Así, el concepto de marca se separa de los demás competidores y establece barreras de entrada a nuevas empresas. Una marca, al final una fuente de ingresos extra, puede convertirse en el activo más importante de una compañía, ya que la lealtad está asociada a la marca. La estrategia de segmentación del mercado y la diferenciación del producto son aspectos determinantes para las marcas (Moore y Reid, 2008), especialmente para las que buscan financiación mediante crowdfunding (Fondevila-Gascón et altri, 2015).

"El posicionamiento parte del producto, aunque va más allá de éste y analiza el posicionamiento en la mente de los clientes potenciales" (Ries y Trout, 1990, p. 18).

Para un adecuado posicionamiento hay que diseñar una oferta para ocupar un puesto distintivo en la mente y maximizar el beneficio (Serralvo y Tadeu, 2005). Esto guía la estrategia de marketing, identifica los objetivos y clarifica la esencia de la marca. Ello resalta una cualidad del producto y lo diferencia de los competidores a partir de sus atributos o beneficios, siendo estos relevantes y proveer de beneficios al cliente, además de ser entregados de una forma más eficiente que la competencia. Existen elementos que favorecen la asociación en la mente de los consumidores, generando un buen posicionamiento (Pérez, 2012) es decir nivel de asociación o fortaleza del vínculo entre la marca y su atributo, lo que viene determinado por las experiencias; nivel de atractivo la relevancia que el consumidor adjudica a cierto atributo del producto o marca y nivel de diferenciación o diferencia marca-atributo que es percibida por el consumidor en comparación a otras marcas.

Las estrategias de posicionamiento dependen de la empresa. Cada una se plantea objetivos y necesidades diferentes. Dependiendo de estas enfatizará en características distintivas que la hagan sobresalir frente a sus competidores para así ser más atractivas en el mercado. El posicionamiento responde a un proceso analítico basado en cuatro cuestiones (Kapferer, 2008); el ¿para qué?: beneficio tácito que nuestro producto o marca ofrece al consumidor; el ¿para quién?: público objetivo claro y definido; el ¿por qué?: elementos que hacen que se necesite el producto; $y$ ¿contra quién?: competencia directa que puede llegar a la mente del consumidor. La estrategia de posicionamiento pretende atraer la atención del mercado 
y quedarse en el top of mind del consumidor (Sharot, 2017). Un posicionamiento eficaz es aquel que no se puede imitar a corto plazo (Aaker, 1991) gracias a características como el atributo, el beneficio racional y psicológico y la calidad o precio relativo.

A efectos de posicionar existen estrategias de uso o aplicación que se enfocan en el uso puntual o determinado del producto, de usuario en el sentido de asociar la marca con un perfil de usuario en específico, como por ejemplo utilizar a un influencer o celebrity para ligar a un segmento con la marca o producto y de competencia la cual genera ventajas competitivas comparadas con las de los competidores. Como es poco habitual poderse posicionar como la mejor marca, si se es líder es decir el que alcanza primero la mente del consumidor; también se puede ser seguidor, buscar un hueco en gama de producto o en una zona geográfica.

\subsection{La generación millennial y el consumo}

La generación que surge después de los años 80 y alcanza su etapa adulta después del año 2000 es la millennial. Esta es la generación sucesora a los baby boomers, nacidos a final de la segunda guerra mundial y la llamada generación $\mathrm{X}$, nacidos entre los años 60 y 80 . Esta generación toma lugar a finales del siglo XX, después de dos guerras mundiales y numerosas crisis económicas. Nacen en una época de aparente estabilidad económica, pero justo al entrar en el nuevo siglo se ven envueltos en tiempos revueltos: en menos de 10 años han sufrido una crisis económica global, los estragos del terrorismo, las guerras y pandemias víricas como el coronavirus. En 2020 representaban el 75\% de la fuerza laboral del mundo $\mathrm{y}$ es lo que hace que pongamos nuestras miradas en ellos como futuros consumidores y usuarios. Es un segmento en crecimiento con nuevas características, necesidades y demandas en el entorno digital (Fondevila-Gascón et altri, 2020).

Las tres generaciones anteriormente mencionadas, tienen formas distintas de ver la vida e incorporarse a ella, lo que causa que sus expectativas, objetivos y formas de pensar o actuar e incluso comprar, se distingan. Es por ello que es importante profundizar en sus características y visión, para entender y predecir su comportamiento.

El factor con el que más se identifica a los millennials es la tecnología, gracias al salto del entorno analógico al digital. Aunque esa generación no considera que la tecnología sea definitiva, admite que les permite forjar su identidad (Fondevila-Gascón y Carreras-Alcalde, 2010). La tecnología es percibida como esencial para ampliar horizontes, comunicarse y establecer relaciones interpersonales, desde su origen (Fondevila-Gascón, 2004). Los millennials admiten que la tecnología les ha otorgado el poder de cambiar el mundo positivamente desde sus dispositivos móviles, generando movilizaciones y corrientes sociales (Fondevila-Gascón et altri, 2015b).

En este sentido, los millennials experimentan una realidad que mezcla los mensajes de comunicación digitales y analógicos. Esto provoca que las empresas creen estrategias de comunicación omnicanal, lo que supone que la empresa está en varios canales a la vez y puede cambiar de canal. Se ha comprobado que en la toma de decisión de compra (Fondevila-Gascón, Carreras-Alcalde y Del Olmo-Arriaga, 2012).

Esta disponibilidad de terminales explica la evolución hacia el consumidor 2.0, especialista en comprar en movilidad, con capacidad de encontrar ofertas, opiniones e información 
de un producto antes de proceder a la compra y con habilidades gracias al triple play y al quíntuple play (Fondevila-Gascón, 2009).

Hay que tener en cuenta que está creciendo el porcentaje de españoles que busca y se informa sobre sus productos en la web antes de comprarlos físicamente en tienda desde el $52 \%$ en el 2014 (Nielsen, 2014).

Se puede catalogar al target millennial como muy crítico y exigente (Fondevila-Gascón et altri, 2015c). De hecho, un elevado porcentaje de consumidores dejaría de comprar los productos de una marca a raíz de una experiencia de compra negativa. Para los millennials, la experiencia de compra y la personalización pueden alcanzar más valor que el mismo producto en sí. Es por ello que las empresas deben centrarse en el cliente como eje estratégico mediante herramientas como el Customer Relationship Management (CRM) y el Social CRM, para conocer más al cliente, satisfacer sus necesidades reales y generar satisfacción y predisposición a repetir la compra con la marca. De ahí surge el engagement y la fidelización.

Curiosamente, en indicadores intangibles los millennials se muestran satisfechos y se consideran felices, sentimientos reforzados por ideas de libertad y capacidad de expresión. Esta generación está orgullosa de su nacionalidad (83\%), pero al mismo tiempo tiene curiosidad por el mundo (88\%), lo que explica que el producto más buscado y comprado en línea por los millennials en España sean tickets de avión, creciendo 15 puntos en comparación al año anterior (Observatorio Cetelem España, 2017).

Los millennials se sitúan entre los principales consumidores de moda. Según datos de Acotex (Asociación Empresarial del Comercio Textil), tras una caída de las ventas en 2007, el sector de la moda en España experimentó una tendencia positiva que culminó en un gran incremento de las ventas en el ejercicio de 2014. En 2015, las ventas experimentaron un descenso hasta volver a los niveles del 2013. En 2016 el sector textil se recuperó hasta 2020, aún no se han equiparado los volúmenes de ventas registrados durante los años anteriores a la crisis. De hecho, la facturación del sector textil en España cayó un 2,27\% en 2018 (Acotex, 2019). En algunas temporadas el cambio climático y las consiguientes elevadas temperaturas suponen un menor gasto en prendas invernales. La caída de las ventas también es un efecto colateral de la inestabilidad política en Cataluña, convertida uno de los problemas que más preocupan a los españoles según el CIS. Indudablemente, los Black Friday y las campañas de Navidad impulsan el consumo y lograron mejorar los datos anuales.

En el ámbito retail de moda las ventas son muy competidas a causa del comportamiento del consumo, caracterizado por la incertidumbre y la volatilidad, y de la mayor oferta comercial, acumulada en la fase de recuperación de la recesión económica. Cataluña es la comunidad autónoma española que más factura, seguida por Madrid. Las tres provincias con mayor gasto medio anual en ropa son Madrid (547,12 €), Baleares $(535,66 €)$ y Navarra $(528,28 €)$. Por otro lado, las provincias con menor gasto en el sector textil son Extremadura $(354,38 €)$ y Galicia $(390,1 €)$. 


\section{Metodología}

El objetivo general de la investigación consiste en conocer el posicionamiento de las principales marcas que comercializan moda en España, Zara, H\&M, Louis Vuitton y Gucci, en la mente de los millennials españoles. Los objetivos específicos son los siguientes: determinar qué atributos se relacionan más con cada marca; determinar los atributos que se consideran fundamentales; determinar la fidelidad de los millennials hacia las marcas; determinar la valoración de la percepción general de las marcas; identificar los motivos de la percepción en las valoraciones de las marcas; determinar cuáles son las marcas con más notoriedad Top of mind; y determinar la satisfacción de los clientes de las diferentes marcas.

El criterio de elección de las marcas es la facturación en el ejercicio en el que se recogen los datos para la investigación y el ranking Best Global Brands. Se trata de empresas caracterizadas por su adaptabilidad y flexibilidad (Martínez, 2007).

Las hipótesis planteadas para este trabajo son las siguientes:

H1. Los millennials sitúan a las marcas Zara, H\&M, Louis Vuitton y Gucci en su top of mind. H2. El conocimiento de las marcas Zara, H\&M, Louis Vuitton y Gucci es elevado.

H3. Existe una asociación entre reconocimiento de marca y la posesión de prendas de las marcas.

H4. Los clientes de las marcas de moda presentan una fidelidad elevada.

En cuanto al desarrollo del trabajo de campo, el método de investigación es cuantitativo. Se utiliza la encuesta, una fuente de información primaria.

En cuanto a la elección de las marcas, Interbrand es una empresa consultora que ofrece servicios relacionados con la creación de marcas, con el fin de darles una identidad, propósito y rol para crear una marca que aporte rentabilidad a su empresa (Interbrand, 2019). Para seleccionar a las marcas de las que se analiza el posicionamiento, se recurrió al ranking Best Global Brands de Interbrand, primera metodología de valoración de marcas en contar con un certificado ISO. Se seleccionan cuatro marcas de la industria textil que figuran en este ranking: Louis Vuitton, H\&M, Zara y Gucci, ya que se trata de un sector industrial en crecimiento y fundamental en el PIB de cada país. Además, estas cuatro marcas de ropa cuentan como target con los millennials, el segmento estudiado.

En esta línea, la muestra en el trabajo de campo está compuesta enteramente por este grupo, ya que es un tipo de público relativamente nuevo y con unas características singulares expuestas en el marco teórico. Otro factor de elección de las marcas es que son antagonistas directos en sus respectivas categorías, ya sea low cost, ya sea lujo.

En cuanto al método de recolección de información primaria elegido para elaborar el trabajo de campo y el método de análisis utilizado para analizar los datos extraídos, se recurre a fuentes secundarias tanto internas como externas a la marca para conocer qué atributos y características quieren comunicar a los consumidores. Sin embargo, para conocer qué atributos y otras características atribuyen los millennials residentes en España a las distintas marcas, se recurre a una fuente de información primaria: la encuesta. 
En este caso, la encuesta está conformada por 13 preguntas cerradas, divididas entre sociodemográficas y de posicionamiento. Las preguntas se formulan para cumplir el objetivo de aceptar o rechazar las hipótesis iniciales y de conocer el posicionamiento de las cuatro marcas: Zara, H\&M, Louis Vuitton y Gucci en la mente de los millennials. Es por ello que se eliminan del cuestionario las respuestas de cualquier persona con una edad no comprendida entre los 17 y 37 años. Se consiguió un número total de respuestas de 950.

\section{Resultados}

En cuanto al análisis demográfico, se presentan las características sociodemográficas de la muestra encuestada. En el análisis univariante y bivariante, se presentan los datos relativos al posicionamiento de las marcas obtenidas de la encuesta. Finalmente, se aceptan o rechazan las hipótesis en función de una serie de pruebas estadísticas.

Demográficamente, se observa que, en cuanto al género, la muestra está proporcionalmente correctamente distribuida, ya que está integrada por un $47,2 \%$ de mujeres y un $52,8 \%$ de hombres. La media de edad es de 22,4 años, con una desviación típica de +/-4,39 años. La mayoría de los encuestados son estudiantes (34,9\%), tipología seguida de trabajadores $(31,6 \%)$. También existe un número relevante de personas que estudian y trabajan simultáneamente $(24,1 \%)$.

Los trabajadores manifiestan mayoritariamente estudios de Formación Profesional (FP) $(35,8 \%)$ y universitarios $(33,6 \%)$. Asimismo, los desocupados exponen estudios de FP $(62,5 \%)$ y estudios básicos (25\%). Finalmente, gran parte de los estudiantes está cursando estudios universitarios $(51,4 \%)$ y FP $(26,5 \%)$.

Se observa que tres de las marcas que son objeto de estudio están entre el top 7 del top of mind de marcas más mencionadas por los encuestados (Figura 1). La primera posición la ocupa Zara, con cerca de un 31\% de las respuestas. Gucci se sitúa en el tercer puesto con un 6,49\% de elecciones, y Louis Vuitton concita un exiguo 2,88\% de las respuestas. Estos datos son estadísticamente significativos. Ello implica que Zara, Gucci y Louis Vuitton gozan de notoriedad. En cambio, la empresa que no aparece en el top 7 de la muestra es H\&M, ubicada en una discreta decimoquinta posición. Ello valida parcialmente H1 (Los millennials sitúan a las marcas Zara, H\&M, Louis Vuitton y Gucci en su top of mind.

En referencia al reconocimiento de marcas (Figura 2), se observa que las cuatro marcas elegidas para esta investigación disponen de un elevado grado de reconocimiento, ya que al menos el 95\% de la muestra las ha identificado. Ciertamente, al tratarse de un sector naturalmente atractivo para los jóvenes, y al ser intenso en cuanto a despliegue de campañas de marketing tanto analógicas como digitales, es comprensible esta cifra de reconocimiento de marca. Probablemente en otros sectores menos populares o más especializados los baremos serían menores, de forma que se interpreta que el macro entorno ayuda a incrementar el reconocimiento de las marcas. Ello confirma $\mathrm{H} 2$, el conocimiento de las marcas Zara, H\&M, Louis Vuitton y Gucci es elevado. 


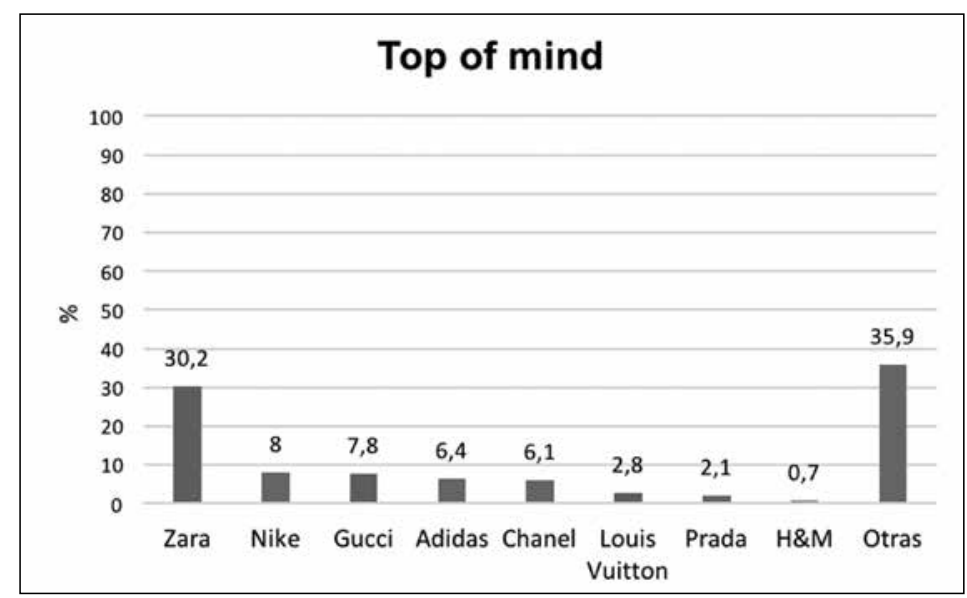

1

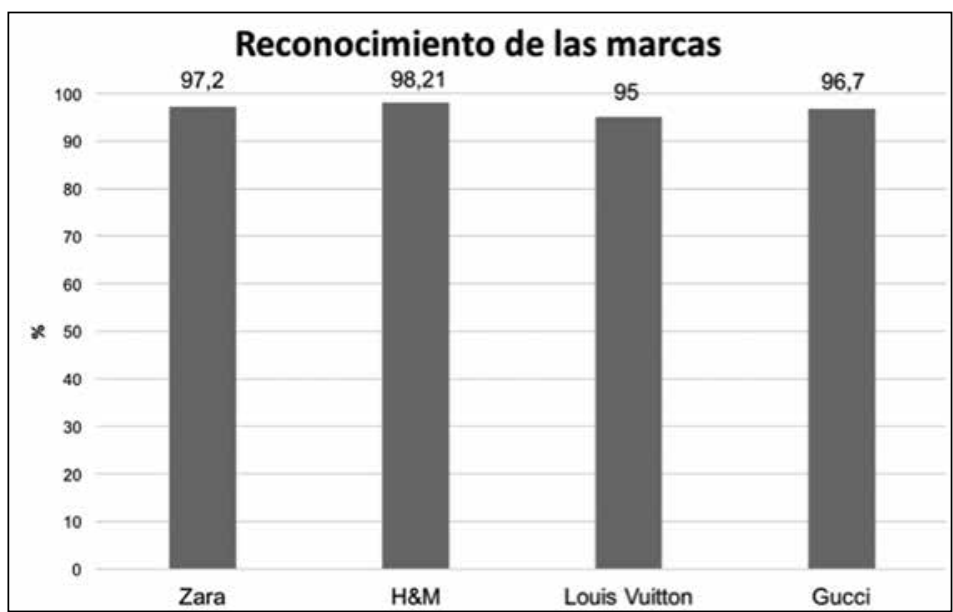

2

Figura 1. Top of mind de las marcas de moda. Fuente: elaboración propia en colaboración con Anna Martorell y Alejandro Garrido. Figura 2. Reconocimiento de las marcas de moda. Fuente: elaboración propia. 


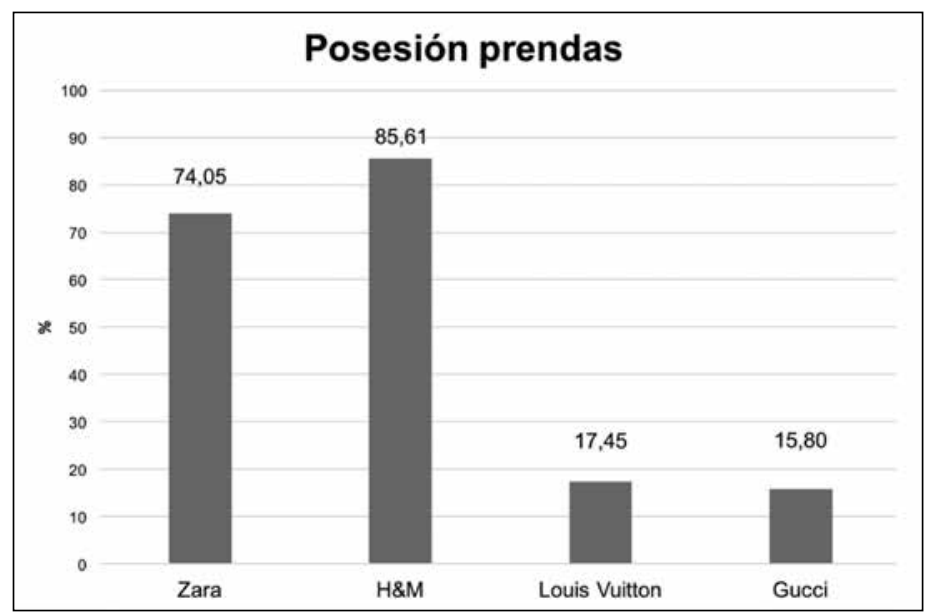

3

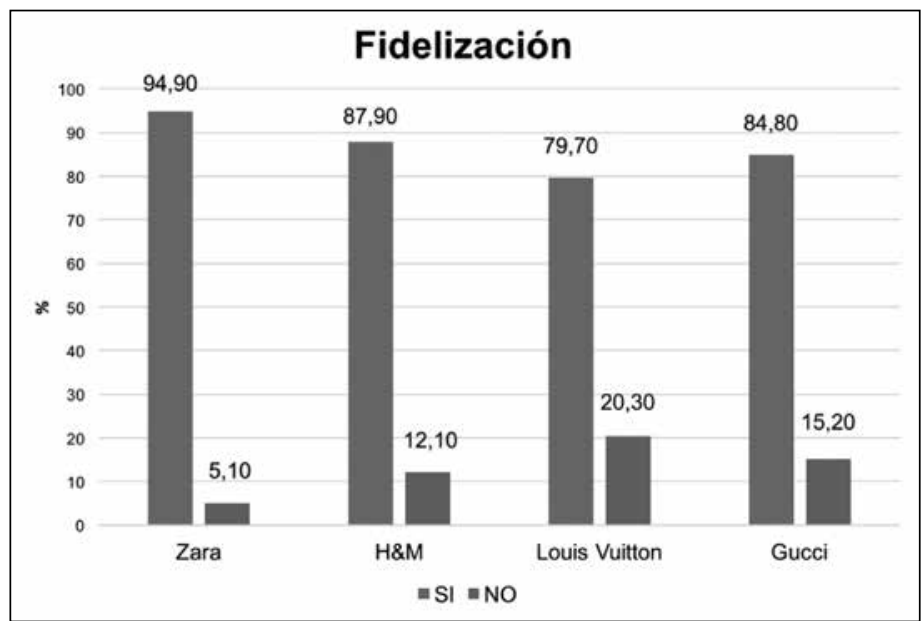

4

Figura 3. Posesión de prendas de las marcas de moda. Fuente: elaboración propia.

Figura 4. Grado de fidelización de las marcas de moda. Fuente: elaboración propia. 
Uno de los indicadores de apego a una marca es su consumo, es decir, la posesión de productos o servicios de la mencionada marca. En este sentido, se observa (Figura 3) que la mayoría de prendas que la muestra manifiesta haber adquirido son de H\&M (en el 85,61\% de casos), seguido por Zara (con un 74,05\%). Estos datos no resultan sorprendentes, ya que estas marcas cuentan como target principal con los encuestados, enfocando gran parte de su estrategia de marketing en ellos. Por otro lado, un 17,45\% de la muestra posee algún producto Louis Vuitton, y un $15,8 \%$ de Gucci. Esos resultados se adaptan a la membresía de un porcentaje de componentes de la muestra, puesto que los clientes de ambas marcas forman parte de un mercado más maduro, aunque sus estrategias se encuentren inmersas en un cambio. Se refuta $\mathrm{H} 3$, porque existe una asociación entre reconocimiento de marca y la posesión de prendas de las marcas.

A los miembros de la muestra que afirmaron que poseían prendas de las distintas marcas se les preguntó si volverían a adquirir prendas de dichas marcas. Esta pregunta pretendía determinar si las marcas cuentan con compradores fieles o no. Zara obtiene los mejores resultados en términos de fidelidad, ya que un 94,9\% de compradores volvería a confiar en la marca, y tan solo un 5,1\% no volvería a hacerlo (Figura 4).

En el caso de H\&M, también se puede afirmar que sus clientes son leales, porque un 87,9\% volverían a comprar sus productos, frente a un $12,1 \%$ que no lo haría. Gucci ocupa la tercera posición, con una fidelidad del $84,8 \%$ de los encuestados. Los consumidores de Louis Vuitton son los menos fieles, aunque el 79,9\% que lo son representa una cifra elevada. Ello confirma $\mathrm{H} 4$, que los clientes de las marcas de moda presentan una fidelidad elevada.

\section{Conclusiones}

En líneas generales, se observa un conocimiento elevado por parte de los millennials de las marcas Zara, H\&M, Louis Vuitton y Gucci en cuanto a top of mind y conocimiento marca, salvo en algún matiz.

La actividad de marketing, sobre todo la digital, que llevan a cabo esas marcas, sobre todo en redes sociales e incluso en soluciones interactivas innovadoras como el HbbTV, son una de las causas de esos índices de conocimiento (Fondevila-Gascón y Carreras-Alcalde, 2010).

Además, se percibe una retroalimentación entre el posicionamiento en la mente de los clientes potenciales (Ries y Trout, 1990) y la fidelidad de los compradores, pero no en cambio si se intenta realizar una asociación entre reconocimiento de marca y la posesión de prendas.

En global, se pueden considerar tres elementos favorecedores de la asociación en la mente de los consumidores en aras de generar un adecuado posicionamiento (Pérez, 2012). Se trata del nivel de asociación, la fortaleza del vínculo de los atributos con los que la marca quiere que el público la asocie, y la asociación real generada en la mente de los consumidores; el nivel de atractivo e importancia que le da el consumidor a cierto atributo o atributos en particular del producto o marca y el nivel de diferenciación o diferencia que percibe el consumidor en comparación a otras marcas. Se observa que los atributos de las 
marcas resultan atractivos, lo que explica la notoriedad, el reconocimiento, la satisfacción, la valoración positiva, los altos índices de posesión y de fidelidad. Se infiere que, para que una marca sea percibida como diferente, debe contar con una serie de ingredientes que le concedan un elevado grado de relevancia, distinción o prioridad, ya que gracias a ellos pueden diferenciarse del resto de marcas con las que rivaliza.

\section{Lista de Referencias Bibliográficas}

Aaker, D. (1991). Managing brand equity: capitalizing on the value of a brand name. New York: The Free Press.

Aaker, D. (1997). Building Strong Brands. New York: The Free Press.

Aaker, D. (2011). Brand Relevance: Making Competitors Irrelevant. San Francisco: Jossey-Bass Aaker, J. (1997). Dimensions of Brand Personality. Journal of Marketing Research, 34(3): 347-356.

Acotex (Asociación Empresarial del Comercio Textil) (2019). Informe sobre el sector textil. Madrid: Acotex.

American Marketing Association (2018). Definition of marketing. Disponible en: https:// www.ama.org/AboutAMA/Pages/Definition-of-Marketing.aspx.

Fondevila-Gascón, Joan-Francesc (2004). "Las redes de telecomunicaciones de cable histórico: realidad y tendencias”. Revista de Comunicación de la SEECI (Sociedad Española de Estudios de la Comunicación Iberoamericana), 11(VII): 67-89.

Fondevila-Gascón, Joan-Francesc (2009). "El peso de la televisión en el triple play de los operadores de cable en España y en Europa”. ZER, Revista de Estudios de Comunicación (Journal of Communication Studies), 14(27), 13-31.

Fondevila-Gascón, Joan-Francesc (2010). El cloud journalism: un nuevo concepto de producción para el periodismo del siglo XXI". Observatorio (OBS ${ }^{*}$ ) Journal, 4(1): 19-35.

Fondevila-Gascón, Joan-Francesc; Carreras-Alcalde, Marta (2010). La tecnologia com a eina al servei de l'educació: una visió humanitzadora. Barcelona: CECABLE.

Fondevila-Gascón, Joan-Francesc (2013). Periodismo ciudadano y cloud journalism: un flujo necesario en la Sociedad de la Banda Ancha. Comunicación y Hombre, 9: 25-41.

Fondevila-Gascón, Joan-Francesc; Carreras-Alcalde, Marta; Del Olmo-Arriaga, Josep-Lluís (2012). Impacto de las tecnologías de la información y la comunicación en la elección de Universidad: el caso de Internet y las redes sociales. Edutec-E. Revista Electrónica de Tecnología Educativa, 39, 1-12.

Fondevila-Gascón, Joan-Francesc; Carreras-Alcalde, Marta; Seebach, Swen; Pesqueira-Zamora, María-Jesús (2015a). "How Elders Evaluate Apps - A Contribution to the Study of Smartphones and to the Analysis of the Usefulness and Accessibility of ICTS for Older Adults". Mobile Media and Communication, 3(2): 250-266.

Fondevila-Gascón, Joan-Francesc; Santana-López, Eva; Rom-Rodríguez, Josep; Mata, Judit; Masip, Pere (2015b). El crowdfunding como fórmula de financiación de proyectos: análisis empírico. Revista Científica Hermes-FIPEN, 14: 24-47. 
Fondevila-Gascón, Joan-Francesc; Berbel, Gaspar; Muñoz, Mònica; Mir-Bernal, Pedro; Puiggròs, Elena (2015c). "Using smartphones reservations for hotels in Barcelona for a business segment: Empirical analysis”. Global Journal of Computer Sciences, 5(2): 60-67.

Fondevila-Gascón, Joan-Francesc; Botey-López, Jordi; Rom-Rodríguez, Josep (2017). Formats emergents en televisió: anàlisi comparativa d'aplicacions publicitàries interactives en HBBTV. Comunicació: Revista de Recerca i d'Anàlisi [Societat Catalana de Comunicació], 34(1): 67-81.

Fondevila-Gascón, Joan-Francesc; Gutiérrez-Aragón, Óscar; Copeiro, Meritxell; Villalba-Palacín, Vicente; Polo-López, Marc (2020). "Influencia de las historias de Instagram en la atención y emoción según el género”. Comunicar. Revista Científica de Comunicación y Educación, 63: 1-10.

Interbrand (2019). Best Global Brands 2018 Ranking. New York: Interbrand.

Kapferer, J. N. (2008). New strategic brand management: creating and sustaining brand equity long term. London y Philadelphia: Kogan Page.

Keller, K. L. (1998). Strategic Brand Management: Building, Measuring, and Managing Brand Equity. Upper Saddle River, New Jersey: Prentice-Hall.

Kotler, P. (2000). Marketing Management Millennium Edition. New Jersey: Prentice- Hall, Inc. Kotler, P. (2001). A Framework for Marketing Management. New Jersey: Prentice- Hall, Inc. Kotler, P. y Keller, K. L. (2000). Marketing Management. New Jersey: Prentice-Hall. Martínez, A.M. (2007). Un modelo de empresa innovadora y flexible: el caso Zara. RIPS: Revista de investigaciones politicas y sociológicas, 1(6): 69-80.

Moore, K., Reid, S. (2008). The Birth of Brand: 4000 Years of Branding History. Munich: McGill University.

Nielsen (2014). Hábitos del e-commerce. Madrid: Nielsen.

Observatorio Cetelem España (2017). Informe del consumo en España. Madrid: Cetelem. Pérez, C. (2012). La esencia del marketing. Madrid: Pérez Carballada.

Ries, A.; Trout, J. (1990). Posicionamiento: La batalla por su mente. Madrid: McGraw-Hill. Serralvo, F. A. y Tadeu, M. (2005). Tipologías del posicionamiento de marcas. Un estudio conceptual en Brasil y España. Revista Galega de Economía, 1-2 (14): 1-15.

Sharot, T. (2017). The Influential Mind: What the Brain Reveals About Our Power to Change Others. Nueva York: Henry Holt and Co.

\footnotetext{
Abstract: Millennials are characterized by living linked to technology and new forms of communication. Through social networks millennials are related to the main brands multiplying the interaction and constantly evolving perceptions with them, a fact that contrasts with other generations that could not relate so quickly and frequently with brands. By studying the perceptions that millennials have about Zara, H\&M, Louis Vuitton and Gucci, we seek to know the positioning of each of these brands among the selected target, spanish millennials, as well as understand the position they occupy among millennials. There is a moderate association between attributes and brand and a high degree of recognition and notoriety.
} 
Keywords: Fashion - millennials - luxury - perception - positioning - tecnology - brands - comunication - social media - identity.

Resumo: A geração do milênio está ligada à tecnologia e a novas formas de comunicação. Por meio das redes sociais, a geração do milênio interage com as principais marcas, multiplicando interação e percepções em constante evolução com elas, fato que contrasta com outras gerações que não conseguiam se relacionar de forma tão rápida e frequente com as marcas. Ao estudar as percepções que os millennials têm sobre Zara, H\&M, Louis Vuitton e Gucci, buscamos conhecer o posicionamento de cada uma dessas marcas entre o target selecionado, os millennials espanhóis, bem como entender a posição que ocupam entre os millennials. Existe uma associação moderada entre atributos e marca e um alto grau de reconhecimento e notoriedade.

Palavras chave: Moda - millennials - luxo - percepção - posicionamento - tecnologia marcas - comunicação - redes sociais - identidade.

[Las traducciones de los abstracts fueron supervisadas por el autor de cada artículo] 